ORGANISATION EUROPÉENNE POUR LA RECHERCHE NUCLÉAIRE (CERN)

Laboratoire Européen pour la Physique des Particules

CERN-PH-EP/2004-006

12-March-2004

\title{
The light sbottom mass window revisited
}

\author{
Patrick Janot \\ CERN, PH Department, CH-1211 Geneva 23, Switzerland \\ e-mail: Patrick.Janot@cern.ch
}

\begin{abstract}
An apparent excess of more than $4 \sigma$ in the $\mathrm{e}^{+} \mathrm{e}^{-} \rightarrow$ hadron cross section for centre-of-mass energies between 20 to $209 \mathrm{GeV}$ has recently been reported. This excess was found to be well reproduced over the whole energy range by the production of a pair of sbottoms with mass $6 \mathrm{GeV} / c^{2}$ and mixing angle $\cos \theta_{\text {mix }}=0.18$. It is shown here that the reported excess essentially vanishes after the implementation of important improvements in the treatment of the PDG data. After these fixes, the combination of PEP, PETRA, TRISTAN and LEP data allows the 95\% C.L. exclusion of a light sbottom decaying hadronically for any mixing angle, if its mass is below $6.0 \mathrm{GeV} / c^{2}$. The light sbottom mass window is closed.
\end{abstract}

Submitted to Physics Letters B 


\section{Introduction}

At the end of the last millenium, the Tevatron Collaborations [1, 2] came out with a bottom quark production cross section at $\sqrt{s}=1.8 \mathrm{TeV}$ in excess of the theoretical prediction by about a factor of two. Refined parton density functions have recently been shown to account for the difference in the recent data recorded at $\sqrt{s}=1.96 \mathrm{TeV}[3]$.

A more exotic model [4, in which a pair of gluinos with mass 12 to $16 \mathrm{GeV} / c^{2}$ is produced in $\mathrm{p} \overline{\mathrm{p}}$ collisions, with subsequent decays into a bottom quark and a sbottom, with mass 2 to $5.5 \mathrm{GeV} / c^{2}$, has been shown to also fit the excess well.. In this model, the sbottom must either be long-lived or decay via R-parity-violating coupling to light quarks, e.g., $\tilde{b} \rightarrow \overline{\mathrm{u}} \overline{\mathrm{s}}$, to comply with various experimental constraints. Long-lived sbottoms have recently been excluded up to masses of $92 \mathrm{GeV} / c^{2}$ by ALEPH [ [ $]$ in direct searches for $\mathrm{e}^{+} \mathrm{e}^{-} \rightarrow \mathrm{q} \overline{\mathrm{q}} \tilde{\mathrm{q}} \overline{\mathrm{q}}$ and $\mathrm{e}^{+} \mathrm{e}^{-} \rightarrow \tilde{\mathrm{q}} \overline{\tilde{\mathrm{q}}}$, but R-parity-violating prompt hadronic decays have not been addressed by the ALEPH analysis.

The measurements of the $\mathrm{e}^{+} \mathrm{e}^{-} \rightarrow$ hadron cross section at centre-of-mass energies in the range 20 to $209 \mathrm{GeV}$ from PEP [6, 7], PETRA [8]-[12, TRISTAN [13]-[17] and LEP [18], have therefore been re-analysed in a recent preprint [19] to search for a consistent excess of hadronic events. An apparent excess of more than $4 \sigma$ over the standard model prediction has been reported. This excess has been found to be consistent with the pair production of light sbottom quarks, $\mathrm{e}^{+} \mathrm{e}^{-} \rightarrow \tilde{\mathrm{b}} \tilde{\mathrm{b}}$, with mass $m_{\tilde{\mathrm{b}}}=6 \mathrm{GeV} / c^{2}$, a mixing angle such that $\cos \theta_{\text {mix }}=0.18$, and R-parity-violating hadronic decays with a $100 \%$ branching ratio.

It is the purpose of this note to show that important drawbacks, listed in Section 2 , affect the public computer-readable files of the hadronic cross section data [20]. Some of these drawbacks also affect the files from the Review of Particle Physics [21], which therefore ought to be corrected to avoid further miunderstanding of these data. In Section 3, it is shown that the production of a pair of light sbottoms with $\cos \theta_{\text {mix }}=0.18$ is excluded by the sole $\mathrm{Z}$ peak data, once these data are adequately interpreted. The results of the fits presented in Ref. [19] are then re-evaluated and discussed in Section [4 after the implementation of the different corrections. The conclusions are given in Section 5 ,

\section{The data before LEP}

In Ref. [19], the data from PEP, PETRA and TRISTAN have been automatically read from the computer files advertized in Ref. [20]. These files were transmitted in 2001 to the Review of Particle Physics [21]. Important drawbacks were found therein and are corrected as explained below.

\subsection{The PETRA data}

Most of the data from MARK J [1] and TASSO [12] were found to be du- or tri-plicated in Refs. 20, 21], because of successive reappraisals with increasing luminosities and improving analysis techniques, thus superseding earlier results. This fact is well advertized 
in a more recent compilation of the same data [22]. The duplicated data were therefore removed from the sample studied here, by keeping only the final - and most accurate - result for each centre-of-mass energy. For each individual measurement, the resulting cross section change is rather small, but the uncertainty increase is not negligible. This last effect is therefore visible on the average of the four PETRA experiments.

\subsection{The TRISTAN data}

About half of the TOPAZ data [14] was published under the form of the ratio $R$ of the effective Born hadronic cross section to the point-like $\mathrm{e}^{+} \mathrm{e}^{-} \rightarrow \mu^{+} \mu^{-}$cross section. The other half [15, 16] was published directly under the form of the effective Born hadronic cross section. In both cases, the result includes a correction that unfolds the effects of initial state radiation (ISR), while still reflecting the running of the fine structure constant, $\alpha_{\mathrm{QED}}(s)$. (This statement is true for all PEP, PETRA and TRISTAN data.) In contrast, it is claimed in Ref. [20] that the original cross section data [15, 16] were corrected for the $\alpha_{\mathrm{QED}}(s)$ running.

This incorrect assumption led the authors of Ref. 20] to modify their computer files accordingly, with the net effect of increasing the corresponding TOPAZ data by $11 \%$. For example, the measured hadronic cross section reported in Ref. [16], $143.6 \pm 1.5 \pm 4.5 \mathrm{pb}$, is found therein to be in agreement with the standard model prediction of $142.2 \mathrm{pb}$, at a centre-of-mass energy of $57.77 \mathrm{GeV}$. This cross section is modified to $160.3 \pm 1.7 \pm 2.9 \mathrm{pb}$ in the files of Ref. [20, leading to a striking $5 \sigma$ excess visible in Figs. 2, 5 and 8 of Ref. [19. Upon request, this unfortunate error has been corrected since in these files [23]. It should be mentioned, however, that this erroneous correction has never affected the computer files of the Review of Particle Physics [21, although they originate from the same authors.

In the process of comparing the different data compilations, it was noticed that some data from VENUS [24, 25] are listed in Ref. [22], but were omitted in Refs. 20, 21] without explanation. Because there is no reason to ignore these data, they were re-incorporated in this note. They tend to further reduce the TRISTAN average cross section measurement. For example, the VENUS data 25] taken at a centre-of-mass energy of $57.77 \mathrm{GeV}$ lead to an effective Born hadronic cross section of $141.8 \pm 1.8 \pm 4.0 \mathrm{pb}$.

\subsection{Consequences of the drawback fixes}

A comprehensive visual comparison of Refs. [20, 21] and [22] indicated no other striking differences between the data compilations and the original publications [6] - 17], 24, 25]. These important drawbacks, however, call for a serious revision of the relevant chapter of the Review of Particle Physics, to avoid further misunderstanding of these data.

The error-free measurements were then clustered in 2-GeV-wide centre-of-mass-energy bins to approximately reproduce the binning of Ref. [19]. The ratio $R$ was averaged in each bin according to the published uncertainties. The corresponding averaged cross sections $\left(\sigma_{\text {had }}^{0}\right)$ and centre-of-mass energy values are displayed in Table 1, The $R$ values found for $\mathrm{PEP} / \mathrm{PETRA}$ were found to agree with those of an earlier combination [9]. 
The effective Born hadronic cross section $\left(\sigma_{\mathrm{th}}^{0}\right)$ predicted by ZFITTER [26], i.e., the hadronic cross section corrected for trivial QED effects (ISR, $\mathrm{e}^{+} \mathrm{e}^{-}$vertex loops) is also shown in Table 1. Because early TRISTAN data [13, 14, 15, 17, 24] have also been corrected for other electroweak effects in the original publications (with a $\left(m_{\mathrm{top}} / m_{\mathrm{Z}}\right)^{2}$ and a $\log \left(m_{\mathrm{H}} / m_{\mathrm{Z}}\right)$ dependence, and determined with obsolete top and $\mathrm{Z}$ mass values), these corrections have been unfolded here for a sound comparison with the ZFITTER prediction, which includes electroweak effects. The latest TRISTAN data [16, 25] were, more adequately, corrected for QED effects only. The electroweak effect correction need therefore not be unfolded in that case.

Table 1: The ratio $R$ and the effective Born hadronic cross section, $\sigma_{\text {had }}^{0}$, from PEP, PETRA, TRISTAN, as a function of the centre-of-mass energy $(\sqrt{s})$, averaged in 2-GeV-wide centre-of-mass-energy bins. The uncertainties are obtained as the quadratic sum of the averaged statistical and systematic errors. The hadronic cross section predicted by ZFITTER, $\sigma_{\mathrm{th}}^{0}$, is also shown. The last column displays the number of measurements used for each entry.

\begin{tabular}{|c|c|c|c|c|}
\hline \hline$\sqrt{s}(\mathrm{GeV})$ & Ratio $R$ & $\sigma_{\text {had }}^{0}(\mathrm{pb})$ & $\sigma_{\text {th }}^{0}(\mathrm{pb})$ & $N_{\mathrm{pts}}$ \\
\hline \hline 21.995 & $3.86 \pm 0.08$ & $756.8 \pm 14.9$ & 763.2 & 5 \\
25.004 & $4.05 \pm 0.19$ & $615.4 \pm 28.7$ & 592.2 & 3 \\
28.882 & $3.94 \pm 0.07$ & $451.4 \pm 7.7$ & 446.0 & 5 \\
30.607 & $3.89 \pm 0.12$ & $396.8 \pm 11.9$ & 398.3 & 5 \\
34.452 & $4.01 \pm 0.05$ & $323.7 \pm 3.7$ & 316.9 & 13 \\
36.005 & $3.86 \pm 0.12$ & $286.0 \pm 8.6$ & 291.4 & 4 \\
38.224 & $3.88 \pm 0.12$ & $255.4 \pm 7.6$ & 260.4 & 3 \\
41.443 & $4.08 \pm 0.08$ & $228.8 \pm 4.5$ & 224.7 & 10 \\
43.817 & $4.04 \pm 0.05$ & $202.7 \pm 2.7$ & 203.8 & 8 \\
46.049 & $4.22 \pm 0.11$ & $192.0 \pm 5.0$ & 187.6 & 6 \\
53.163 & $4.51 \pm 0.12$ & $154.9 \pm 4.0$ & 153.4 & 12 \\
56.461 & $4.92 \pm 0.11$ & $149.6 \pm 3.4$ & 145.4 & 9 \\
58.067 & $4.95 \pm 0.09$ & $142.4 \pm 2.5$ & 143.2 & 16 \\
60.184 & $5.40 \pm 0.13$ & $144.6 \pm 3.5$ & 142.2 & 9 \\
61.856 & $5.42 \pm 0.18$ & $137.5 \pm 4.5$ & 143.1 & 5 \\
\hline \hline
\end{tabular}

The ratio and the difference of these measured cross sections and those predicted by ZFITTER are displayed in Fig. 1 as a function of the centre-of-mass energy. This figure can be directly compared to Figs. 5 and 8 of Ref. [19]. It can be noticed that most of the excesses shown therein are essentially wiped out. It was also checked that, without the fixes explained above, the figures of Ref. [19] could be reproduced quite faithfully, up to residual binning differences, as shown in Fig. 2. 
Section 2 fixes included
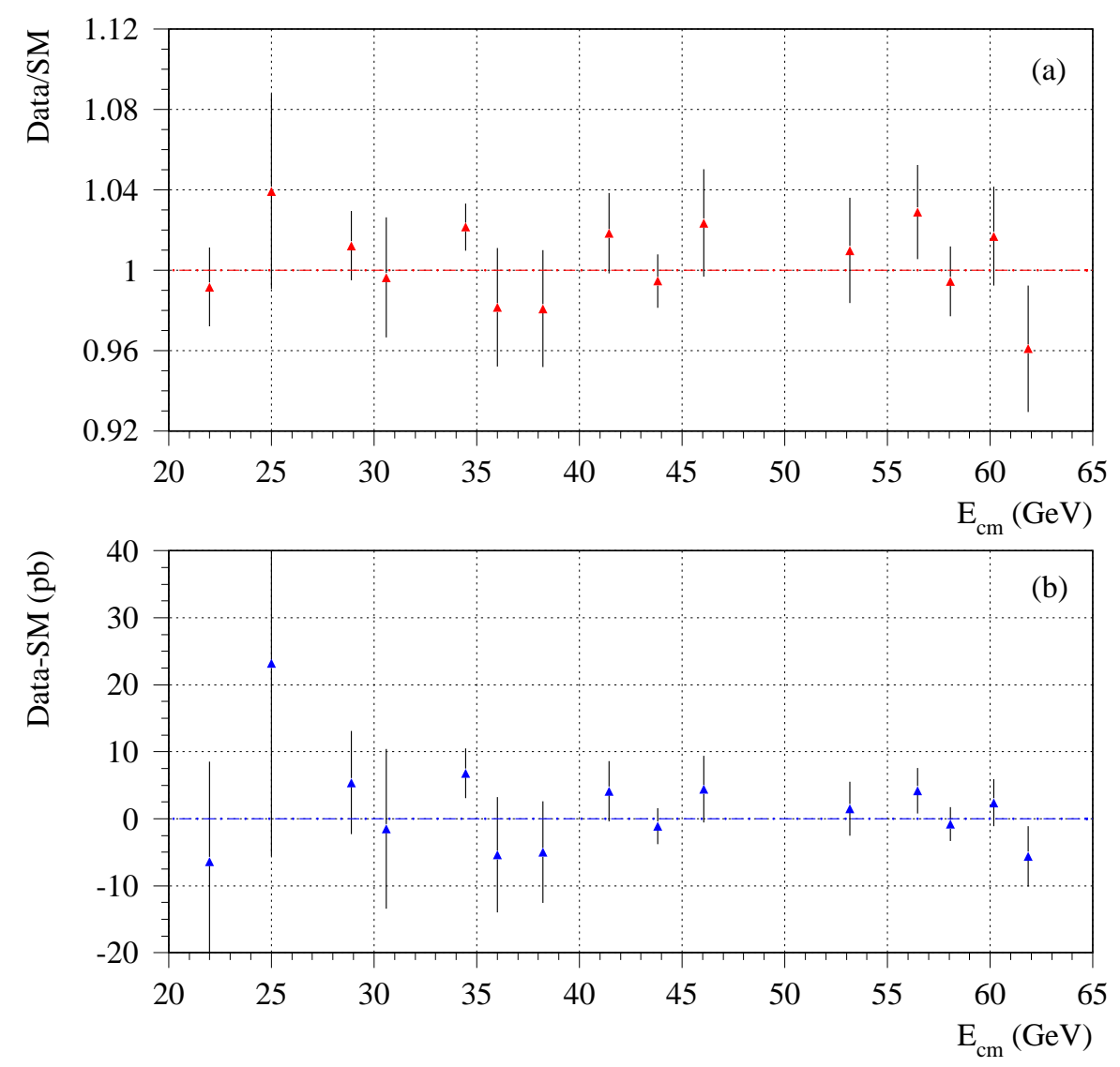

Figure 1: Ratio (a) and difference (b) of the effective Born hadronic cross section measurements and the ZFITTER prediction as a function of the centre-of-mass energy, for PEP, PETRA and TRISTAN data, rebinned as suggested in Ref. [19]. The dash-dotted line indicates the standard model prediction.

\section{A correction in the $\mathrm{Z}$ peak data interpretation}

In Ref. [19], it is claimed that "it is well known that the hadronic cross section at the $Z$ peak, $\sigma_{\text {had }}^{0}$, slightly exceeds the standard model fit". Indeed, the measured value of $\sigma_{\text {had }}^{0}$ is found to be $41.540 \pm 0.037 \mathrm{nb}$, from a fit of the $\mathrm{Z}$ lineshape, to be compared to the standard model prediction of $41.478 \mathrm{nb}$. This comparison is interpreted in Ref. [19] as a slight excess of hadronic events in $\mathrm{Z}$ decays with a cross section of $62 \pm 37 \mathrm{pb}$.

This interpretation is, however, incorrect. First, a larger value of $\sigma_{\text {had }}^{0}$ is instead the sign of a deficit of hadronic $\mathrm{Z}$ decays. The tree-level peak cross section, $\sigma_{\text {had }}^{0}$, is given by

$$
\sigma_{\text {had }}^{0}=\frac{12 \pi}{m_{\mathrm{Z}}^{2}} \frac{\Gamma_{\mathrm{ee}} \Gamma_{\mathrm{had}}}{\Gamma_{\mathrm{Z}}^{2}},
$$



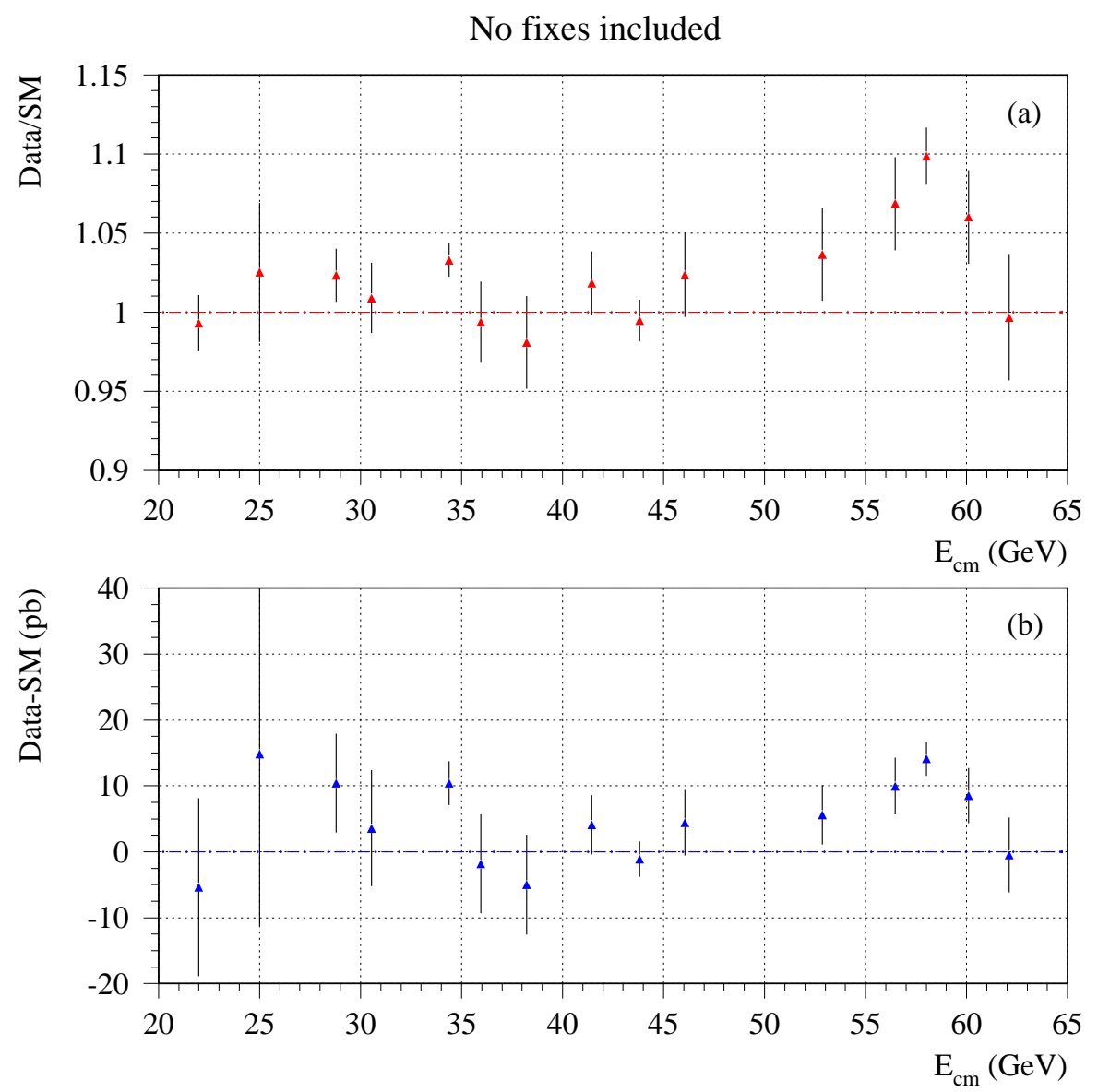

Figure 2: Ratio (a) and difference (b) of the effective Born hadronic cross section measurements and the ZFITTER prediction as a function of the centre-of-mass energy, for PEP, PETRA and TRISTAN data, if the corrections described in Section 2 are not implemented.

where $\Gamma_{\text {ee }}, \Gamma_{\text {had }}$ and $\Gamma_{\mathrm{Z}}$ are the partial $\mathrm{Z}$ decay widths into $\mathrm{e}^{+} \mathrm{e}^{-}$and into hadrons, and the total $\mathrm{Z}$ decay width, respectively. An additonal contribution to the hadronic decays of the $\mathrm{Z}$ would increase $\Gamma_{\text {had }}$, but would also increase $\Gamma_{\mathrm{Z}}^{2}$ twice faster, with a net reduction of $\sigma_{\text {had }}^{0}$. (The observation that the peak cross section decreases with additional contributions to the $\mathrm{Z}$ decays allowed the precise determination of the number of light neutrino species.)

Second, most of the $\mathrm{Z}$ observables would actually be modified in case of an additional (New Physics) contribution to the $\mathrm{Z}$ decays. Let $\varepsilon_{\mathrm{NP}}^{\text {had }}$ be the ratio of this new partial width $\Gamma_{\mathrm{NP}}$ to the total decay width of the $\mathrm{Z}$ without this new contribution. As was shown in Ref. [27, the Z total width $\Gamma_{\mathrm{Z}}$, the peak cross section $\sigma_{\text {had }}^{0}$ and the ratio $R_{\ell}$ of the hadronic to the leptonic branching fractions are modified as follows,

$$
\begin{array}{cll}
\Gamma_{\mathrm{Z}} & \longrightarrow \Gamma_{\mathrm{Z}}\left(1+1.00 \varepsilon_{\mathrm{NP}}^{\mathrm{had}}\right), & {\left[\Gamma_{\mathrm{Z}}+\Gamma_{\mathrm{NP}}\right]} \\
R_{\ell} \longrightarrow R_{\ell}\left(1+1.43 \varepsilon_{\mathrm{NP}}^{\mathrm{had}}\right), & {\left[\left(\Gamma_{\mathrm{had}}+\Gamma_{\mathrm{NP}}\right) / \Gamma_{\ell}\right]} \\
\sigma_{\text {had }}^{0} \longrightarrow \sigma_{\mathrm{had}}^{0}\left(1-0.57 \varepsilon_{\mathrm{NP}}^{\mathrm{had}}\right) . & {\left[\frac{12 \pi}{m_{\mathrm{Z}}^{2}} \frac{\Gamma_{\mathrm{ee}}\left(\Gamma_{\mathrm{had}}+\Gamma_{\mathrm{NP}}\right)}{\left(\Gamma_{\mathrm{Z}}+\Gamma_{\mathrm{NP}}\right)^{2}}\right]}
\end{array}
$$


In Ref. [27], the new hadronic decay channel considered was flavour-democratic. The individual branching fractions into the different quark flavours were therefore not modified. In the case of a sbottom pair production with hadronic R-parity violating decays into light quarks exclusively, the ratio of the $\mathrm{b} \overline{\mathrm{b}}$ branching ratio to the hadronic branching ratio, $R_{\mathrm{b}}$, is also modified according to

$$
R_{\mathrm{b}} \longrightarrow R_{\mathrm{b}}\left(1-1.43 \varepsilon_{\mathrm{NP}}^{\mathrm{had}}\right), \quad\left[\Gamma_{\mathrm{b} \overline{\mathrm{b}}} /\left(\Gamma_{\mathrm{had}}+\Gamma_{\mathrm{NP}}\right)\right]
$$

while $\left(g_{V} / g_{A}\right)$ remains untouched.

Third, these observables would also be modified by the virtual corrections arising from the New Physics responsible for the additional hadronic contribution. As in Ref. [27, the value of $\varepsilon_{\mathrm{NP}}^{\text {had }}$ was fitted to the measurement of the five observables together with the contribution of these virtual effects. The result is

$$
\varepsilon_{\mathrm{NP}}^{\mathrm{had}}=(-0.56 \pm 0.80) \times 10^{-3},
$$

which corresponds to an additional hadronic contribution of

$$
\sigma_{\text {had }}^{\mathrm{NP}}=-24 \pm 36 \mathrm{pb}
$$

instead of the $+62 \pm 37 \mathrm{pb}$ advocated in Ref. 19]. It allows a 95\% C.L. upper limit of $56 \mathrm{pb}$ to be set on the cross section, at the $\mathrm{Z}$ peak, of any additional hadronic contribution to the $\mathrm{Z}$ decays into light quarks only. The resonant contribution of the sbottom pair

production cross section [28] with $m_{\tilde{\mathrm{b}}}=6 \mathrm{GeV} / c^{2}$ and $\cos \theta_{\text {mix }}=0.18$ (the best fit of Ref. [19]) amounts to $\sim 74 \mathrm{pb}$ at the $\mathrm{Z}$ peak. It is therefore excluded at more than $95 \%$ C.L.

\section{Reappraisal of the fit results and discussion}

The techniques of Ref. [19] were applied with no modification to the corrected data files, to see whether the three striking conclusions of the report, i.e.,

1. the overall fit result is consistent with the light sbottom pair cross section;

2. the overall fit result is not disfavoured in any of the regions; and is statistically consistent with the individual fit result in each region;

3. all centre-of-mass energies contribute to the significance of the effect;

are still valid for $m_{\tilde{\mathrm{b}}}=6 \mathrm{GeV} / c^{2}$, and to re-evaluate the significance of the "apparent excess" once the fixes of Sections 2 and 3 are implemented. Not surprisingly, all the results of Ref. [19] could be reproduced if the uncorrected inputs were fed into the fit algorithm.

The four centre-of-mass energy regions were, however, slightly redefined here so as to group together the data from the same collider, and therefore potential common systematic uncertainties, (1) PEP and PETRA; (2) TRISTAN; (3) LEP 1; and (4) LEP 2. This change has no consequence on the overall result. 


\subsection{Result without common systematic uncertainties}

As a first test, the combined negative log-likelihood of Eq. 8 of Ref. 1,

$$
\mathcal{L}=\frac{1}{2} \sum_{i, j=1}^{N} \Delta_{i} S_{i j}^{-1} \Delta_{j} \text { with } \Delta_{i}=\sigma_{\text {had }, i}^{0}-\left[\sigma_{\text {th }, i}^{0}+\alpha \sigma_{\mathrm{NP}, i}^{0}\left(m_{\tilde{\mathrm{b}}}, \cos \theta_{\text {mix }}\right)\right],
$$

where $S$ is the covariance matrix of the $N$ measurements, was determined as a function of the mixing angle in the sbottom sector and of an arbitrary normalization constant $\alpha$ of the sbottom pair production cross section, $\sigma_{\mathrm{NP}, i}^{0}$. The experimental correlations in $S$ are those given in Ref. [18] for the LEP 2 measurements, and were determined as suggested in Ref. 9] for Regions 1 and 2. No other common systematic uncertainties are included in this subsection. For $\alpha=1$ and $m_{\tilde{\mathrm{b}}}=6 \mathrm{GeV} / c^{2}$, the result is displayed in Fig. 通 as a function of $\cos \theta_{\text {mix }}$, before and after the implementation of the corrections.
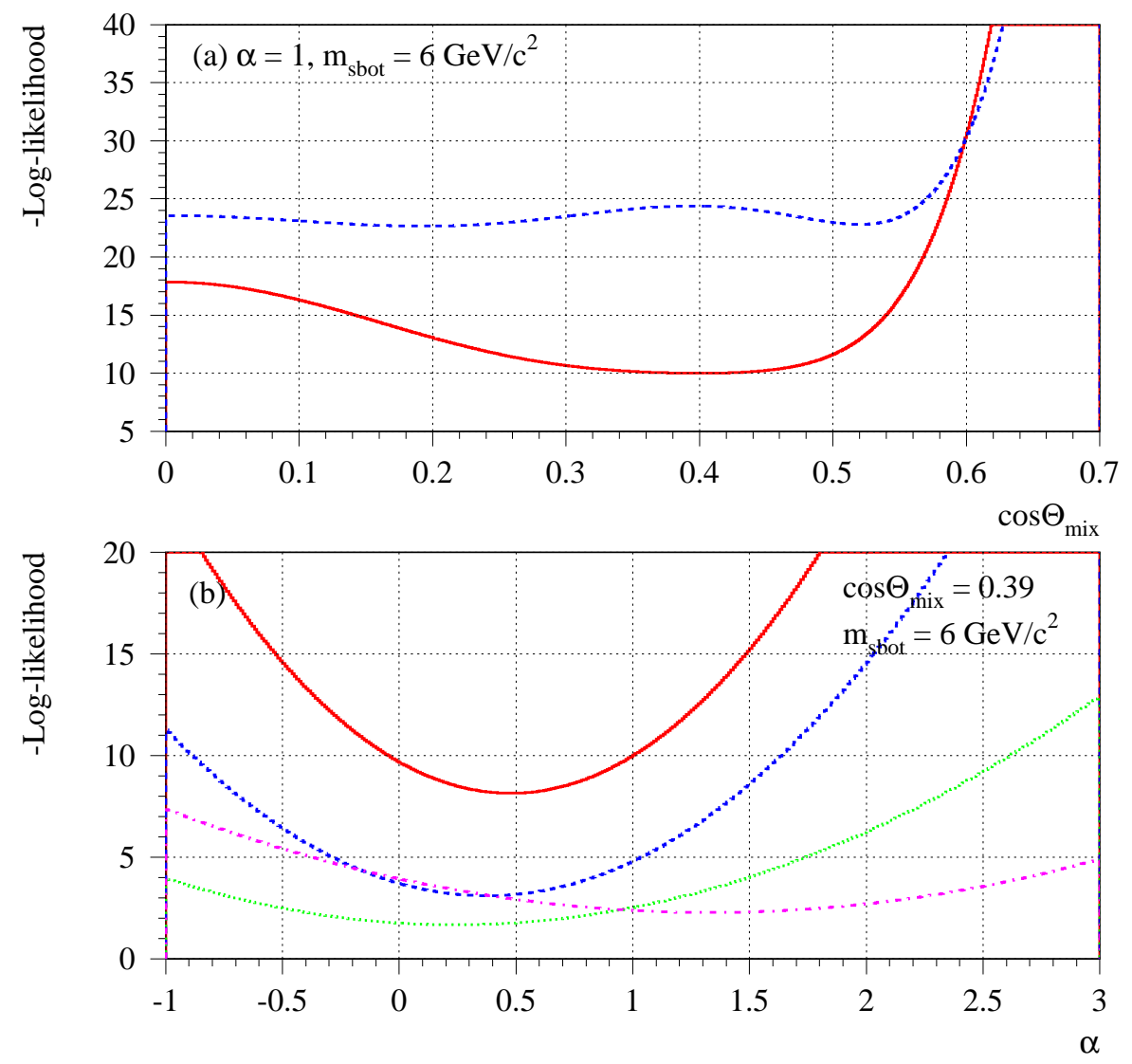

Figure 3: The negative $\log$-likelihood with $m_{\tilde{\mathrm{b}}}=6 \mathrm{GeV} / c^{2}$ (a) as a function of $\cos \theta_{\text {mix }}$ for $\alpha=1$, after (full curve) and before (dashed curve) the correction implementation; and (b) as a function of $\alpha$ for $\cos \theta_{\text {mix }}=0.39$ (full curve), and in Regions 1 (dashed curve), 2 (dotted curve) and 4 (dash-dotted curve)

The curve of Ref. [19] could be identically reproduced (up to an irrelevant normalization constant), with a shallow minimum at $\cos \theta_{\text {mix }}=0.18$. After the fixes, the minimum moves to, not surprisingly, $\cos \theta_{\text {mix }}=0.39 \pm 0.06$, for which the coupling of the sbottom to the $\mathrm{Z}$ is negligibly small (Section B). For this value of the mixing, the $\mathrm{Z}$ peak data do not contribute positively to any potential effect. The third conclusion of Ref. [19] is therefore no longer valid. 
The value of the mixing angle was therefore fixed to $\cos \theta_{\text {mix }}=0.39$. The combined negative log-likelihood and those in Regions 1, 2 and 4 are shown in Fig. 3b as a function of $\alpha$. (In Region 3, the likelihood does not depend on $\alpha$, because of the vanishing sbottom cross section for $\cos \theta_{\text {mix }}=0.39$.) The values of $\alpha$ for which the different negative $\log$ likelihoods are minimized are indicated in Table 2, together with the corresponding $68 \%$ confidence intervals and the 95\% C.L. upper limits on $\alpha$. (This one-sided upper limit is the $\alpha$ value for which the negative log-likelihood increases by $1.64^{2} / 2$ with respect to the minimum.)

Table 2: The values $\alpha_{\text {min }}$ for which the negative log-likelihood is minimized in Regions 1, 2 and 4, and in the combination, together with the $68 \%$ confidence intervals and the $95 \%$ C.L. upper limits, $\alpha_{95}$.

\begin{tabular}{|c|c|c|}
\hline \hline Region & $\alpha_{\min }$ & $\alpha_{95}$ \\
\hline \hline 1 & $0.36 \pm 0.35$ & 0.92 \\
2 & $0.23 \pm 0.58$ & 1.19 \\
4 & $1.31 \pm 0.75$ & 2.52 \\
All & $0.47 \pm 0.27$ & 0.92 \\
\hline \hline
\end{tabular}

In addition to Region 3, neither Region 1 nor Region 2 favour the sbottom hypothesis $(\alpha=1)$. Both regions are, instead, compatible with the standard model $(\alpha=0)$ within one standard deviation. A slight excess in the LEP 2 data, at the $1.7 \sigma$ level, translates to the combined result. The latter, however, excludes the sbottom hypothesis with $m_{\tilde{\mathrm{b}}}=$ $6 \mathrm{GeV} / c^{2}$ at more than $95 \%$ C.L., when no common systematic uncertainty is included. The first and second conclusions of Ref. [19] are therefore invalidated as well. It was checked that, without the various fixes of Sections 2 and 3 , the absolute minimum of the negative likelihood is found at $\alpha=1.22 \pm 0.20$ for $\cos \theta_{\text {mix }}=0.23$, i.e., about $6 \sigma$ away from the standard model, in agreement with the findings of Ref. [19].

\subsection{Discussion of common systematic uncertainties}

In Ref. [19], the common systematic uncertainties are assumed to entirely arise from the standard model prediction of the $\mathrm{e}^{+} \mathrm{e}^{-} \rightarrow \mathrm{q} \overline{\mathrm{q}}$ cross section. The main sources are therefore (i) the knowledge and the running of the strong coupling constant $\alpha_{S}$; (ii) the running of the electromagnetic coupling constant $\alpha_{\mathrm{QED}}$; and (iii) the theoretical accuracy of the prediction from the ZFITTER program.

In Ref. [19, the $\alpha_{S}$ error is doubled, and the three uncertainties are added linearly for a conservative total of about $0.5 \%$. A more reasonable value of $0.2 \%(0.3 \%)$ below (above) the $\mathrm{Z}$ peak, obtained with the original $\alpha_{S}$ uncertainty and a quadratic combination instead, could be easily defended, as is argued in Ref. [19]. The negative log-likelihood was modified to include this common systematic uncertainty, $\eta_{\mathrm{th}}$, as

$$
\mathcal{L}=\frac{1}{2} \sum_{i, j=1}^{N} \Delta_{i} S_{i j}^{-1} \Delta_{j}+\frac{\rho_{\mathrm{th}}^{2}}{2 \eta_{\mathrm{th}}^{2}} \quad \text { with } \quad \Delta_{i}=\sigma_{\mathrm{had}, i}^{0}-\left[\left(1+\rho_{\mathrm{th}}\right) \sigma_{\mathrm{th}, i}^{0}+\alpha \sigma_{\mathrm{NP}, i}^{0}\right]
$$


and minimized with respect to the theoretical bias $\rho_{\text {th }}$ for each value of $\alpha$. The values of $\alpha$ for which the negative log-likelihood is minimized are indicated in Table 3, together with the corresponding $68 \%$ confidence intervals and the $95 \%$ C.L. upper limits on $\alpha$, for $\eta_{\mathrm{th}}=0.2 \%$ and $0.5 \%$, and for $m_{\tilde{\mathrm{b}}}=6 \mathrm{GeV} / c^{2}$.

Table 3: The values $\alpha_{\min }$ for which the combined negative log-likelihood is minimized for $\eta_{\mathrm{th}}=0.2 \%$ and $0.5 \%$, together with the $68 \%$ confidence intervals and the $95 \%$ C.L. upper limits, $\alpha_{95}$.

\begin{tabular}{|c|c|c|}
\hline \hline$\eta_{\mathrm{th}}$ & $\alpha_{\min }$ & $\alpha_{95}$ \\
\hline \hline $0.2 \%$ & $0.44 \pm 0.29$ & 0.93 \\
$0.5 \%$ & $0.41 \pm 0.38$ & 1.02 \\
\hline \hline
\end{tabular}

The sbottom hypothesis with $m_{\tilde{\mathrm{b}}}=6 \mathrm{GeV} / c^{2}$ is excluded in both configurations, or just about. If a common systematic uncertainty of $0.2 \%$ is to be chosen, however, the non-Gaussian nature of uncertainties of theoretical origin has to be taken into account. For example, the missing higher orders in ZFITTER may turn into a bias of $-0.1 \%, 0 \%$ or $0.1 \%$ with an equal probability. (In fact, the least likely value is certainly $0 \%$, as missing orders are expected to contribute a finite amount to the cross section.) Similarly, the uncertainty on the absolute value of $\alpha_{S}\left(m_{\mathrm{Z}}\right)$ is dominated by theory, and cannot be considered as Gaussian. It is therefore probably more adequate to assume a probability density function as displayed in Fig. 4, i.e., flat between $-\eta_{\mathrm{th}}$ and $+\eta_{\mathrm{th}}$, and with a Gaussian shape outside this interval.

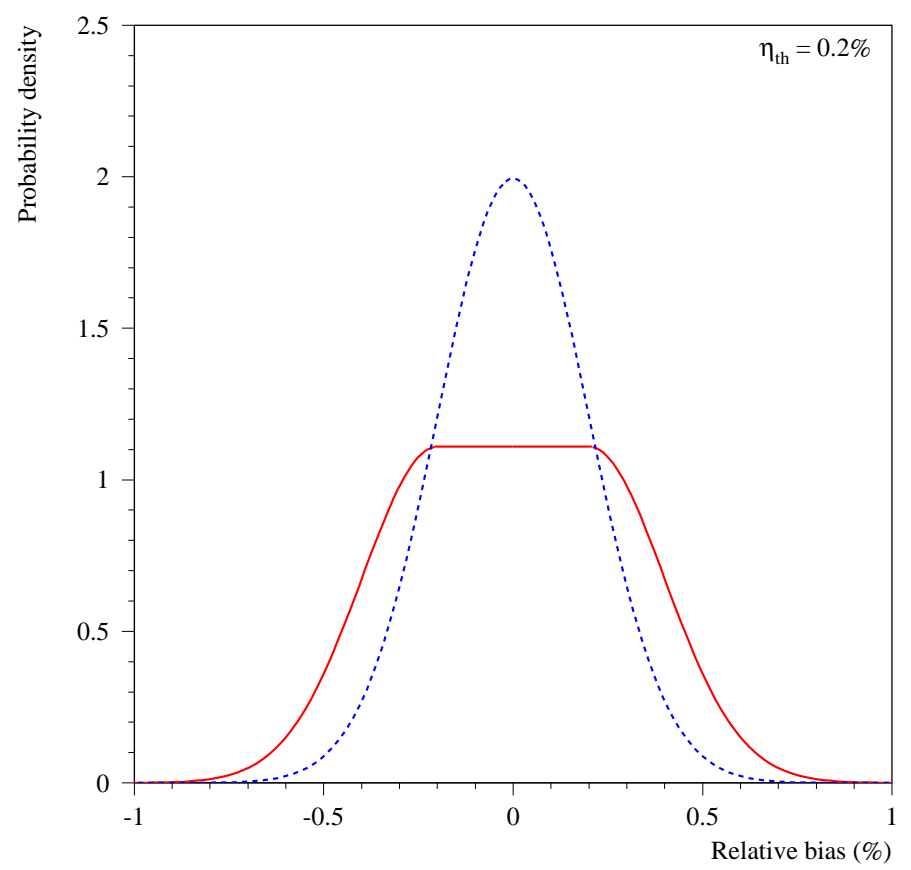

Figure 4: Probability density function for the conventional (Gaussian) systematic uncertainty treatment (dashed curve) and suggested here instead (full curve) to account for the non-Gaussian nature of theory uncertainties, with $\eta_{\mathrm{th}}=0.2 \%$. 
The likelihood was therefore further modified by changing the $\rho_{\mathrm{th}}^{2} / 2 \eta_{\mathrm{th}}^{2}$ term to

$$
\begin{array}{ccr}
\left(\rho_{\mathrm{th}}+\eta_{\mathrm{th}}\right)^{2} / 2 \eta_{\mathrm{th}}^{2} & \text { if } & \rho<-\eta_{\mathrm{th}}, \\
0 & \text { if } & -\eta_{\mathrm{th}}<\rho<\eta_{\mathrm{th}}, \\
\left(\rho_{\mathrm{th}}-\eta_{\mathrm{th}}\right)^{2} / 2 \eta_{\mathrm{th}}^{2} & \text { if } & \rho>\eta_{\mathrm{th}} .
\end{array}
$$

The minimum was found at $\alpha_{\min }=0.35_{-0.29}^{+0.44}$, and the $95 \%$ C.L. upper limit on $\alpha$ was set at 0.98 , thus still excluding the sbottom hypothesis with $m_{\tilde{\mathrm{b}}}=6 \mathrm{GeV} / c^{2}$.

Another possibly important correlated uncertainty, not mentioned in Ref. [19], was also studied here. At the time of PEP, PETRA and TRISTAN, the Monte Carlo programs used to simulate the $\mathrm{e}^{+} \mathrm{e}^{-} \rightarrow \mathrm{q} \overline{\mathrm{q}}$ and $\mathrm{e}^{+} \mathrm{e}^{-} \rightarrow \mathrm{e}^{+} \mathrm{e}^{-}$processes were limited to $\mathcal{O}\left(\alpha_{\mathrm{QED}}\right)$ for the QED corrections. The missing orders have a potential effect on the measured value of $\sigma_{\text {had }}^{0}$ via the prediction of both the hadronic cross section and the Bhabha scattering cross section. Indeed, the former is used to correct the measured $\sigma_{\text {had }}$ for QED effects, and the latter to determine the integrated luminosity. Altogether, the published cross section values have to be corrected as follows,

$$
\sigma_{\text {had }}^{0} \longrightarrow \sigma_{\text {had }}^{0} \times \frac{\sigma_{\text {ee }}^{(\text {all })}}{\sigma_{\mathrm{ee}}^{(1)}} \frac{\sigma_{\text {had }}^{(1)}}{\sigma_{\text {had }}^{(\text {all }}},
$$

where the indices (1) and (all) refer to the cross section prediction up to the QED first order and with all orders available today, respectively. This effect, common to all results published until 1990, has been tentatively estimated by MAC [7] to reduce the ratio $R$ by about $(-1.1 \pm 1.1) \%$. Other experiments have included a 1-to-2\% systematic uncertainty in their result to account for the missing QED higher orders.

With the programs that have been developed for LEP, it is now possible to evaluate this effect with a better accuracy. The $\mathrm{e}^{+} \mathrm{e}^{-} \rightarrow \mathrm{q} \overline{\mathrm{q}}$ and $\mathrm{e}^{+} \mathrm{e}^{-} \rightarrow \mathrm{e}^{+} \mathrm{e}^{-}$cross sections were determined here with and without QED higher orders by ZFITTER with an emulation of the kinematical cuts described in the original publications [6]-[15, [17, 24. It was found that the corrections to Bhabha scattering and hadron production essentially cancel in the ratio of Eq. 13. The remaining contribution of QED higher orders is at the $0.1 \%$ level, almost independently of the event selection and the centre-of-mass energy. The related uncertainties could therefore be reduced, but they were conservatively kept to their published values in this note.

To include this possible additional common bias $\rho_{\exp }$ in the measured $q \bar{q}$ cross section at PEP, PETRA and TRISTAN, the negative log-likelihood was upgraded to

$$
\begin{gathered}
\mathcal{L}=\frac{1}{2} \sum_{i, j=1}^{N} \Delta_{i} S_{i j}^{-1} \Delta_{j}+\frac{\rho_{\mathrm{th}}^{2}}{2 \eta_{\mathrm{th}}^{2}}+\frac{\rho_{\mathrm{exp}}^{2}}{2 \eta_{\exp }^{2}} \\
\text { with } \Delta_{i}=\left(1+\rho_{\mathrm{exp}}^{i}\right) \sigma_{\mathrm{had}, i}^{0}-\left[\left(1+\rho_{\mathrm{th}}\right) \sigma_{\mathrm{th}, i}^{0}+\alpha \sigma_{\mathrm{NP}, i}^{0}\right] .
\end{gathered}
$$

In this expression, $\eta_{\exp }=0.1 \%, \rho_{\exp }^{i}=\rho_{\exp }$ in Regions 1 and 2 (with the exception of the results published by TOPAZ [16] in 1995 and VENUS [25] in 1999, since they contain all the LEP refinements), and $\rho_{\exp }^{i}=0$ in Regions 3 and 4 . The possibly non-Gaussian nature of $\eta_{\text {th }}$ and $\eta_{\text {exp }}$ was dealt with as indicated in Eqs. (10) to (12). 


\subsection{Results}

This negative log-likelihood was then minimized with respect to $\rho_{\text {th }}$ and $\rho_{\exp }$ simultaneously, for each value of $\alpha$, with Gaussian and non-Gaussian uncertainties. The result is displayed in Fig. [ 5 as a function of $\alpha$ in the two configurations, for $\cos \theta_{\text {mix }}=0.39$ and $m_{\tilde{\mathrm{b}}}=6 \mathrm{GeV} / c^{2}$. The values of $\alpha$ for which the negative log-likelihood is minimized are indicated in Table 4, together with the corresponding $68 \%$ confidence intervals and the $95 \%$ C.L. upper limits on $\alpha$. It can be seen that the upper limit on $\alpha$ depends very little on the way the common systematic uncertainties are dealt with. The most conservative approach, with non-Gaussian uncertainties, is chosen here to derive the final results.

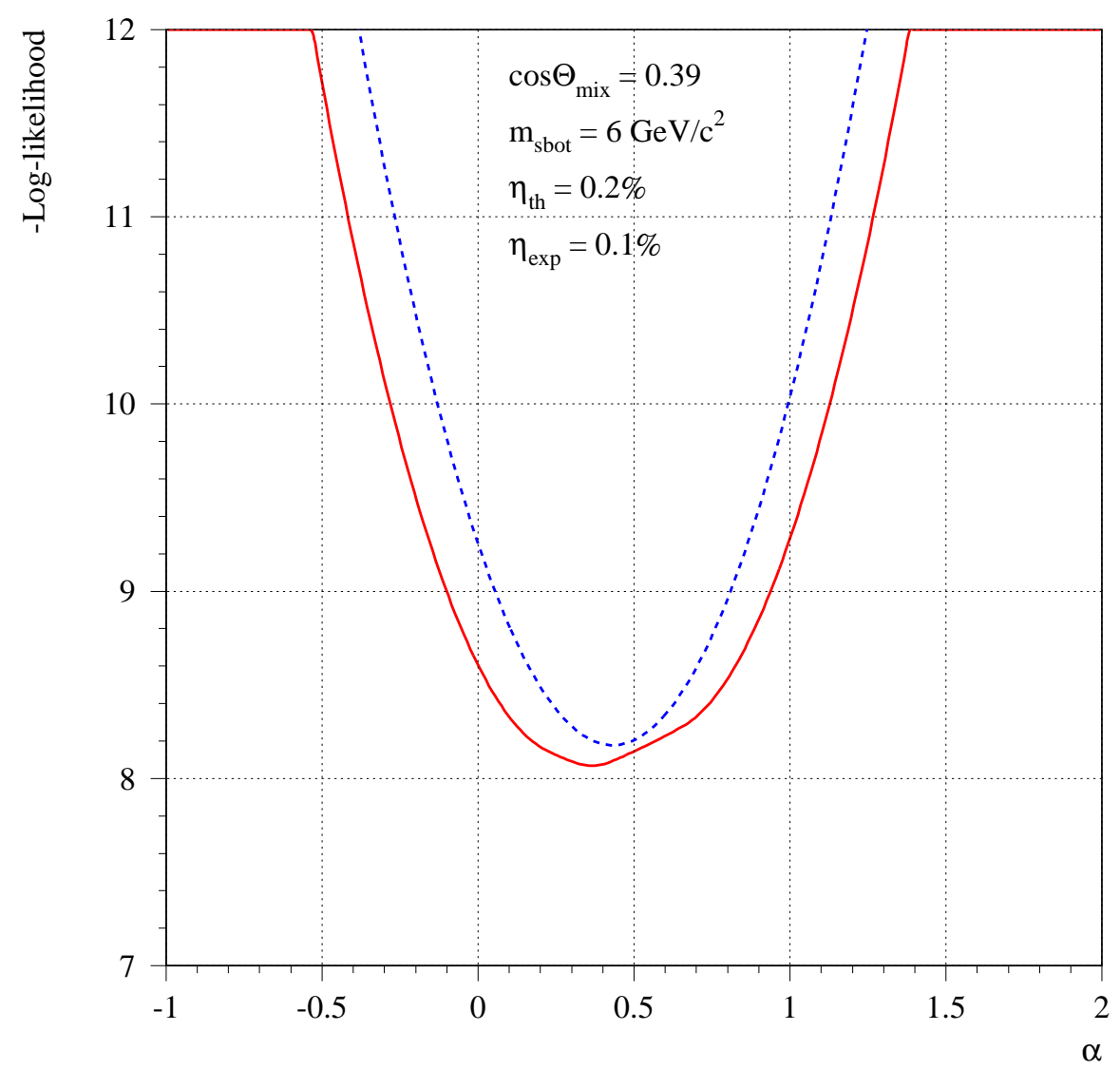

Figure 5: The combined negative log-likelihood curves with all systematic uncertainties included, assumed to be Gaussian (dashed curve) or non-Gaussian (full curve), for $\cos \theta_{\text {mix }}=0.39$ and $m_{\tilde{\mathrm{b}}}=$ $6 \mathrm{GeV} / c^{2}$.

Table 4: The values $\alpha_{\min }$ for which the combined negative log-likelihood is minimized for Gaussian and non-Gaussian uncertainties, together with the $68 \%$ confidence intervals and the $95 \%$ C.L. upper limits, $\alpha_{95}$. The fit results in Regions 1,2 and $4\left(\alpha_{1}, \alpha_{2}\right.$ and $\left.\alpha_{4}\right)$ are also given.

\begin{tabular}{|c|c|c|c|c|c|}
\hline \hline Uncertainties & $\alpha_{\min }$ & $\alpha_{95}$ & $\alpha_{1}$ & $\alpha_{2}$ & $\alpha_{4}$ \\
\hline \hline Gaussian & $0.43 \pm 0.29$ & 0.92 & $0.30 \pm 0.38$ & $0.15 \pm 0.68$ & $1.54 \pm 0.90$ \\
Non-Gaussian & $0.36_{-0.34}^{+0.46}$ & 1.00 & $0.16_{-0.38}^{+0.67}$ & $0.02_{-0.68}^{+0.93}$ & $1.72 \pm 0.90$ \\
\hline \hline
\end{tabular}


The same procedure was repeated by varying the sbottom mass from 0 to $12 \mathrm{GeV} / c^{2}$. For each mass, the $95 \%$ C.L. upper limit on $\alpha$ was determined as explained above. A sbottom with a given mass is excluded if this upper limit is smaller than unity. Figure 6 shows the $95 \%$ C.L. upper limit on $\alpha$ for $\cos \theta_{\text {mix }}=0.39$ as a function of the sbottom mass, with Gaussian and non-Gaussian uncertainties. (In the latter configuration, the non-Gaussian nature of the likelihood was taken into account in the determination of the limit.) Sbottom masses below $6 \mathrm{GeV} / c^{2}$ are excluded at the $95 \%$ confidence level.

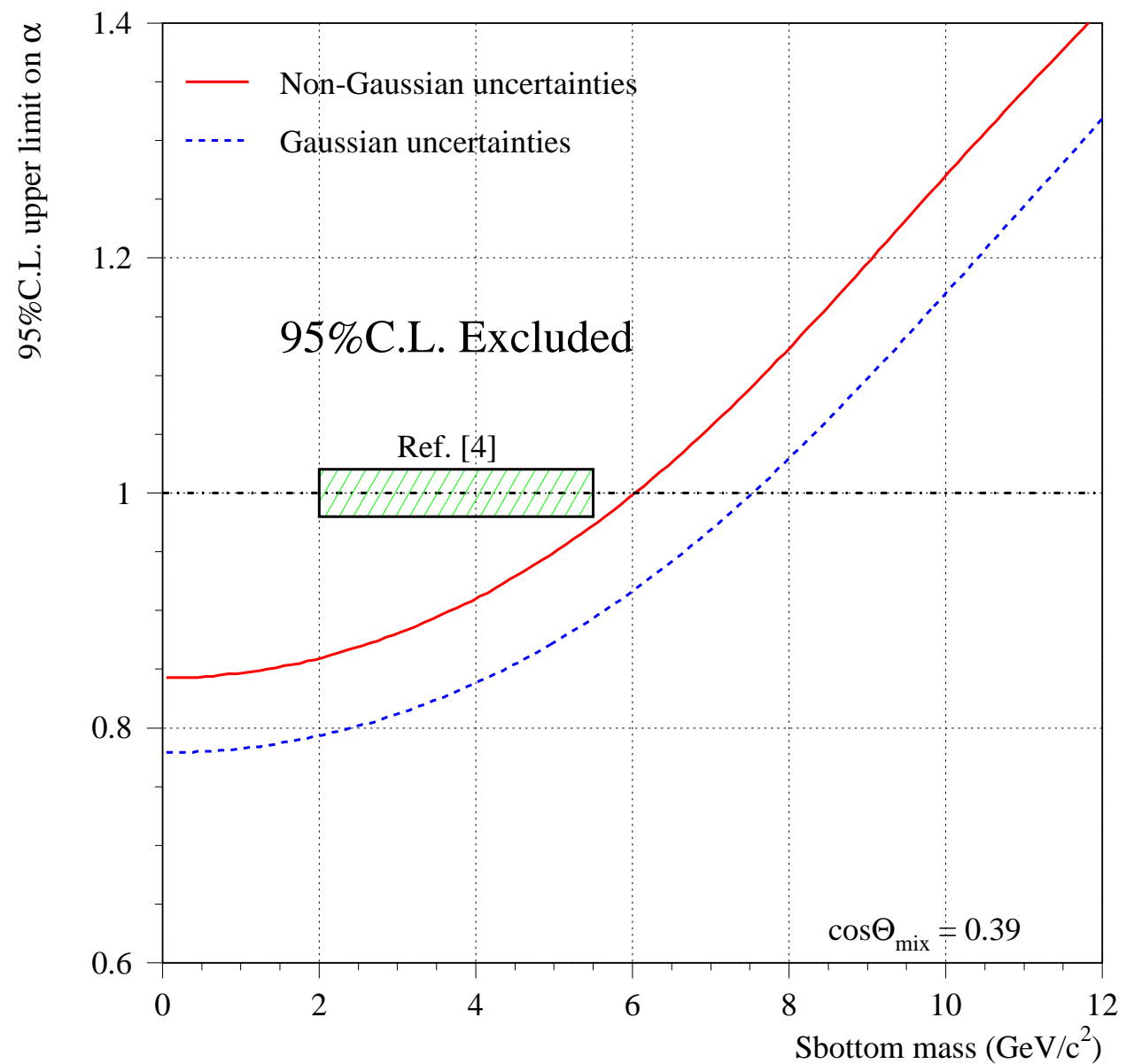

Figure 6: The 95\% C.L. upper limit on $\alpha$ as a function of the sbottom mass, with non-Gaussian (full curve) and Gaussian (dashed curve) common systematic uncertainties. Also shown are the predictions of the model of Ref. 4, now excluded by this analysis.

Because $\cos \theta_{\text {mix }}$ is very much constrained by the $\mathrm{Z}$ peak data, the upper limit on $\alpha$ is expected to be smaller than that shown in Fig. 6] for any other value of the mixing angle. As a check, the procedure was repeated again by varying $\cos \theta_{\text {mix }}$ from 0 to 1 , with non-Gaussian uncertainties. The resulting sbottom mass lower limit is shown in Fig. 7 as a function of $\cos \theta_{\text {mix }}$, and exceeds indeed $6 \mathrm{GeV} / c^{2}$ over the whole range.

It is worth mentioning that the presence of a light sbottom would slow down the running of $\alpha_{S}$ with the centre-of-mass energy. (It would be even more so with an additional light gluino.) Starting from the value accurately measured in $\tau$ decays [29], (the only measurement not affected by a sbottom heavier than $2 \mathrm{GeV} / c^{2}$, and corresponding to $\alpha_{S}\left(m_{\mathrm{Z}}\right)=0.121 \pm 0.003$ in the standard model), this slower running would lead to values of 


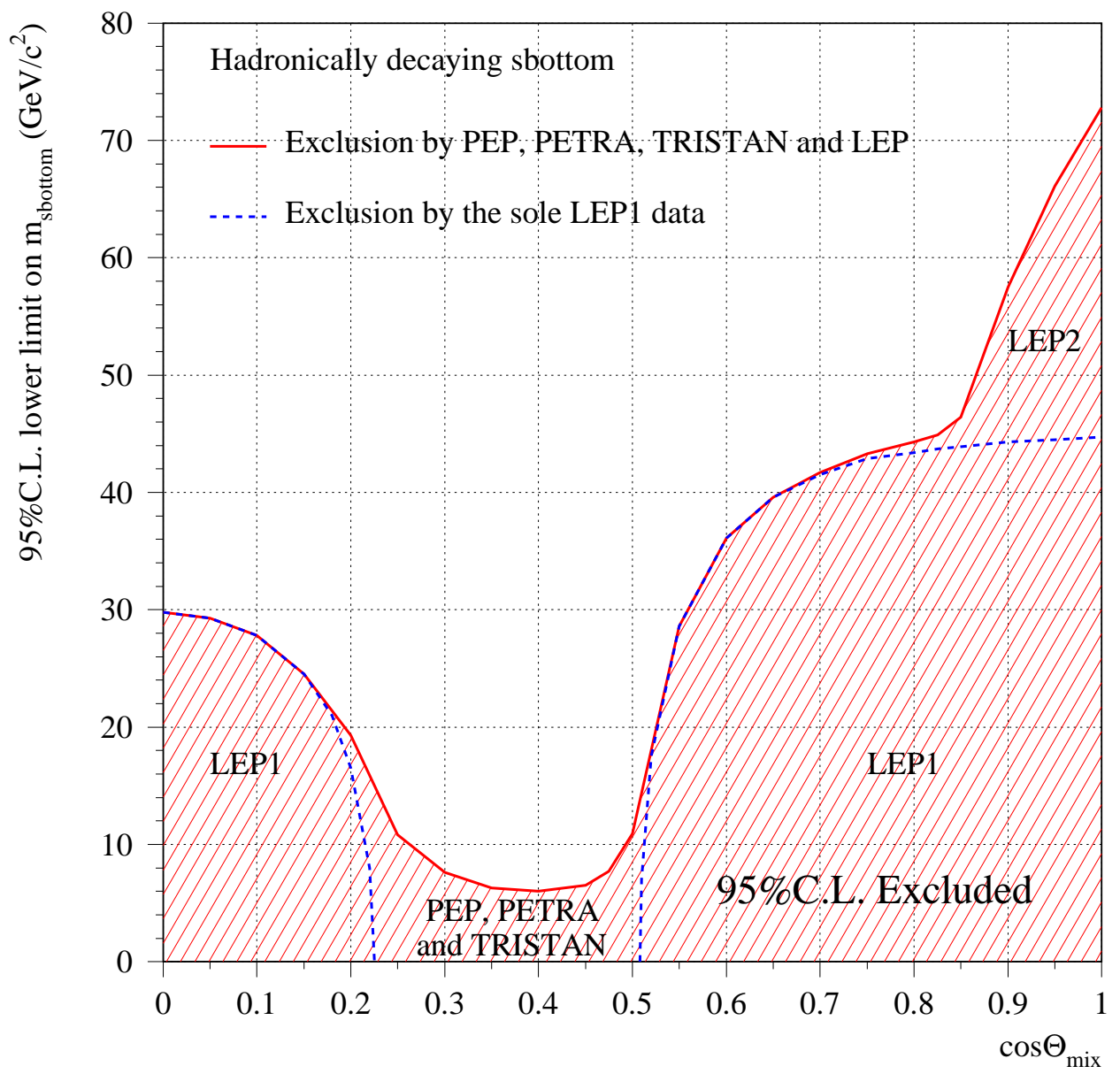

Figure 7: Absolute 95\% C.L. lower limit on $m_{\tilde{\mathrm{b}}}$ as a function of $\cos \theta_{\mathrm{mix}}$, for hadronically decaying sbottoms. The hatched area is excluded at $95 \%$ C.L. The dashed line shows the exclusion achieved with the sole $\mathrm{Z}$ peak data. The region excluded by LEP 2 data at large values of $\cos \theta_{\text {mix }}$ is probably overoptimistic, as four-jet events - expected from such heavy sbottom pair as well as $\mathrm{W}$ pair production are rejected from the q $\bar{q}$ event samples selected above the WW threshold.

$\alpha_{S}$ larger than assumed in this note, at all centre-of-mass energies. The total New Physics contribution (from the direct sbottom production and the increase of $\alpha_{S}$ ) would further increase the total hadronic cross section expected at PEP, PETRA, TRISTAN and LEP. The $6 \mathrm{GeV} / c^{2}$ lower limit on the sbottom mass is therefore probably very conservative.

\section{Conclusion}

The $\mathrm{e}^{+} \mathrm{e}^{-} \rightarrow$ q $\overline{\mathrm{q}}$ excess presented in Ref. [19] is indeed only "apparent". When important improvements in the treatment of the compiled data are implemented, the excess vanishes to one standard deviation or thereabout. Altogether, the PEP, PETRA, TRISTAN and LEP data allow a light sbottom decaying hadronically to be excluded at 95\% C.L. for any mixing angle, if its mass is below $6.0 \mathrm{GeV} / c^{2}$. When combined with the result of Ref. [5] in which a stable sbottom with mass below $92 \mathrm{GeV} / c^{2}$ is excluded, this analysis definitely invalidates the model of Ref. 4] with a $12-16 \mathrm{GeV} / c^{2}$ gluino and a $2-5.5 \mathrm{GeV} / c^{2}$ sbottom. 


\section{Acknowledgments}

I am grateful to German Rodrigo, Sabine Kraml and the CERN Journal Club on Phenomenology for pointing the article of Ref. [19] to me, and to Daniel Treille, Günther Dissertori and Michelangelo Mangano for interesting discussions. Many thanks are due to Bolek Pietrzyk, Helmut Burkhardt, Gerardo Ganis and Brigitte Bloch for their help with ZFITTER and other Monte Carlo programs, and for many enlightening exchanges.

Above all, I would like to warmly thank Michael Schmitt for his accurate remarks and his collaborative help in the analysis of the important drawbacks affecting the public computer files of the hadronic cross section data.

\section{References}

[1] D0 Coll., Phys. Rev. Lett. 74 (1995) 3548;

D0 Coll., Phys. Rev. Lett. 85 (2000) 5068;

D0 Coll., Phys. Lett. B 487 (2000) 264.

[2] CDF Coll., Phys. Rev. Lett. 71 (1993) 500;

CDF Coll., Phys. Rev. Lett. 71 (1993) 2396;

CDF Coll., Phys. Rev. D 50 (1994) 4252;

CDF Coll., Phys. Rev. Lett. 75 (1995) 1451;

CDF Coll., Phys. Rev. D 65 (2002) 052005;

CDF Coll., Phys. Rev. D 66 (2002) 032002.

[3] M. Cacciari et al., "QCD analysis of first b cross section data at 1.96 TeV", hep-ph/0312132, CERN-TH/2003-298 (2003), and references therein.

[4] E.L. Berger, Phys. Rev. Lett. 86 (2001) 4231.

[5] ALEPH Coll., Eur. Phys. J. C 31 (2003) 327.

[6] MARK II Coll., Phys. Rev. D 43 (1990) 34.

[7] MAC Coll., Phys. Rev. D 31 (1985) 1537.

[8] CELlO Coll., Phys. Lett. B 144 (1984) 297.

[9] CELLO Coll., Phys. Lett. B 183 (1987) 400.

[10] JADE Coll., Phys. Rep. 148 (1987) 67.

[11] MARK J Coll., Phys. Lett. B 85 (1979) 463;

MARK J Coll., Phys. Rep. 63 (1980) 337;

MARK J Coll., Phys. Lett. B 108 (1982) 63;

MARK J Coll., Phys. Rev. D 34 (1986) 681. 
[12] TASSO Coll., Phys. Lett. B 83 (1979) 261;

TASSO Coll., Z. Phys. C 4 (1980) 87;

TASSO Coll., Phys. Lett. B 113 (1982) 499;

TASSO Coll., Z. Phys. C 22 (1984) 307;

TASSO Coll., Phys. Lett. B 138 (1984) 441;

TASSO Coll., Z. Phys. C 47 (1990) 187.

[13] AMY Coll., Phys. Rev. D 42 (1990) 1339.

[14] TOPAZ Coll., Phys. Lett. B 234 (1990) 525.

[15] TOPAZ Coll., Phys. Lett. B 304 (1993) 373.

[16] TOPAZ Coll., Phys. Lett. B 347 (1995) 171.

[17] VENUS Coll., Phys. Lett. B 198 (1987) 570; VENUS Coll., Phys. Lett. B 246 (1990) 297.

[18] The LEP Colls. and the LEPEWWG, "A combination of preliminary electroweak measurements and constraints on the standard model", hep-ex/0312023, and references therein.

[19] M. Schmitt, "Apparent excess in $e^{+} e^{-} \rightarrow$ hadrons", hep-ex/0401034, v2 (2004).

[20] O.V.Zenin et al., "A compilation of total cross section data on $e^{+} e^{-} \rightarrow$ hadrons and pQCD tests", hep-ph/0110176 (2001) - unpublished.

Computer-readable files can be found at http://wwwppds.ihep.su:8001/comphp.html

[21] Review of Particle Physics, Phys. Rev. D 66 (2002) 259.

Computer-readable files can be found at http://pdg.1bl.gov/2002/contents_plots.html

[22] M.R. Whalley, J. Phys. G: Nucl. Part. Phys. 29 (2003) A1.

[23] O.V.Zenin, private communication.

[24] VENUS Coll., Phys. Lett. B 234 (1990) 382.

[25] VENUS Coll., Phys. Lett. B 447 (1999) 167.

[26] D. Bardin et al., Comput. Phys. Commun. 133 (2001) 229.

The version 6.36 of ZFITTER was used here, with FOT2 set to -1 and CONV set to 0 , to compute the effective Born hadronic cross section corrected for QED effects, $\sigma_{\text {had }}^{0}$, with the most recent vacuum polarization determination from http://www-zeuthen.desy.de/ fjeger/hadr5n.f.

[27] P. Janot, Phys. Lett. B 564 (2003) 183.

[28] H. Eberl, A. Bartl and W. Majerotto, Phys. Lett. B 349 (1995) 463.

[29] ALEPH Coll., Phys. Lett. B 307 (1993) 209;

OPAL Coll., Eur. Phys. J. C 7 (1999) 571. 